\title{
Evaluación de satisfacción a los estudiantes sobre el uso del software Microsoft Teams
}

\author{
Sarauz Mateo ${ }^{1}$, Shuguli Jorge ${ }^{2}$, Vaca David ${ }^{3}$ y Villafuerte Rita ${ }^{4}$ \\ \{mateo.sarauz, David.shugulí, David.vaca, rita.villafuerte\}@udla.edu.ec \\ https://orcid.org/0000-0001-7559-012X ${ }^{1}$, https://orcid.org/0000-0002-3655-76152, \\ https://orcid.org/0000-0003-2853-420X ${ }^{3}$, https://orcid.org/0000-0001-5016-129X4. \\ Universidad de las Américas \\ Quito-Ecuador
}

Recibido (01/07/20), Aceptado (17/07/20)

Resumen: El uso de las Tecnologías de la información y la comunicación ha permitido introducir mejoras en la forma como se desarrollan los procesos de enseñanza y aprendizaje, así como los procesos propios a la gestión y administración de las actividades académicas. Sin embargo, el índice de adopción de estas herramientas es reducido en cobertura y en profundidad. No obstante, para el uso de la herramienta Teams hay que detallar las ventajas y desventajas encontradas y finalizar con conclusiones en base a las experiencias personales.

Palabras Clave: Software; Microsoft Teams; Herramienta; Ventajas; Experiencia.

\section{Assessment of student satisfaction on the use of Microsoft Teams software}

\begin{abstract}
The use of Information and Communication Technologies has led to improvements in the way teaching and learning processes are developed, as well as the processes of their own to the management and administration of academic activities. However, the adoption rate of these tools is reduced in coverage and in depth. However, for the use of the Teams tool you must detail the advantages and disadvantages found and conclude with conclusions based on personal experiences.
\end{abstract}

Keywords: Software; Microsoft Teams; Tool; Advantages; Experience. 


\section{I.INTRODUCCIÓN}

Como objetivo principal, la finalidad de este estudio fue conocer la satisfacción de los estudiantes sobre el uso de Microsoft Teams para lo cual se realizó una encuesta compuesta de 17 ítems realizada a 55 personas y que arrojo un alfa de Cronbach de 0.92 , lo que permite garantizar una alta confiabilidad en el manejo de los datos y en el procesamiento de la información.

Este trabajo está compuesto por cuatro secciones, la primera fue una encuesta dirigida a una comunidad exclusivamente de estudiantes que manejan o utilizan este tipo de plataformas para su aprendizaje. Se realizaron varias preguntas entre las cuales la que más resaltó fue la pregunta de la importancia de que los recursos de audio y video se fusionen de manera rápida y eficaz a lo que un $71,4 \%$ de los encuestados respondieron que consideran muy importante que la plataforma funcione corridamente en cuanto audio y video. Dentro de la encuesta de satisfacción también se realizó la pregunta dentro de la encuesta acerca de la importancia del tiempo de configuración del software para adaptarse a sus gustos y necesidades a lo que un $42,9 \%$ de los encuestados respondieron que es muy importante, realmente un porcentaje bajo en comparación a las demás preguntas realizadas que tuvieron una acogida de mayores porcentajes, con lo que se plantea la siguiente interrogante los estudiantes no buscan un software adecuado a sus necesidades? O quizá los estudiantes no consideran importante la necesidad de adaptarse a una plataforma educativa online, pues, como lo mencionamos anteriormente la plataforma no hace al estudiante, sino el estudiante hace a la plataforma se piensa que no existe la necesidad de satisfacción de que al estudiante le gusta la herramienta, sino que solamente se adapte y conozca la funcionalidad de dicha plataforma.

En la educación virtual el problema no es la herramienta, sino el uso que hacemos de ella [1]. Con esta frase queda en evidencia que sin bien es cierto, la herramienta no forma al estudiante, sino que el estudiante forma a la herramienta, por lo que planteamos la idea de escoger o de preferir herramientas colaborativas e interactivas lo que justifica quizá el contenido de la plataforma Microsoft Teams, llevando así la transformación de clases presenciales a un formato online calificando como aceptable las medidas tomadas por la institución. Por lo tanto, el profesorado y el estudiantado tienen que colaborar para dar una respuesta que integre decisiones metodológicas y tecnológicas, a la vez que garantice la equidad, la seguridad y transparencia para todos los actores externos e internos.

\section{DESARROLLO}

Las primeras escuelas virtuales se desarrollaron en Australia, Nueva Zelanda, América del Norte y el Reino Unido, generalmente en áreas donde la baja densidad de población hacía difícil la escolarización por medios tradicionales. El desarrollo de la informática consolida la utilización de ordenadores con fines educativos, dando lugar a la creación de diferentes escuelas virtuales alrededor de todo el mundo tradicionalmente, las instituciones que ofrecen educación a través de la red han tenido al uso de plataformas educativas que ofrecen al alumno una gama de servicios tales como repositorios de documentación, foros, correo, etc., en un entorno cerrado, accesible mediante nombre de usuario y contraseña, y con un funcionamiento uniforme. Este tipo de educación ha experimentado un notable crecimiento en el Ecuador y más aún en este tiempo en el que estamos sumergidos en una pandemia mundial [1].

Hoy en día, la tecnología es un factor que juega un papel de vital importancia dentro de la sociedad, ya que en este tiempo en el que nos encontramos en una pandemia la educación y el trabajo son aspectos que no pueden ser dejados de lado en nuestro país. Con el fin de brindar una educación virtual de calidad, existen plataformas en las que se puede desarrollar o recibir clases y realizar teletrabajo. Es por ello por lo que existen estas plataformas digitales como lo son: Zoom, Google Meet, y Microsoft Teams; esta última será la herramienta que nosotros analizaremos a medida que se va desarrollando este articulo con el fin de conocer si en realidad los estudiantes sienten que su funcionalidad es ideal para el uso educativo.

En el Ecuador se encuentran infinidad de herramientas web 2.0, dentro de ellas se encuentran plataformas para colaboración, manejo de curso, organización de clases, creación de videos, generadores de cuestionarios, gestionadores de tareas, lecciones y exámenes, entre muchas otras. Para la mayoría de la población valora más las aplicaciones que son gratuitas y apropiadas para el ambiente educativo e idealmente que no requieran descarga en la computadora. Entre las herramientas más usadas se encontraron Edmodo que es una excelente herramienta para trabajo colaborativo ya que se puede crear grupos, asignar tareas y gestionar el progreso de los alumnos, esta herramienta está integrada con Google Apps. Microsoft, Une Note y Office. Animoto es una herramienta muy útil para crear y compartir video al mismo tiempo permite agregar texto donde los estudiantes y tutores no deberían tener ninguna dificultad. Educarplay es una herramienta creativa e interactivas que usan profesores de manera 
interactiva presentaciones, pruebas y juegos. YouTube muchos docentes usan esta plataforma para cargar sus clases de manera pública que ayuden a los estudiantes a comprender mediante la repetición de los videos, el proyecto YouTube EDU es español es un espacio educativo abierto y gratuito que te permitirá completar las clases con contenidos educativos de alta calidad, otras de las plataformas usadas en Ecuador es Zoom, esta herramienta generalmente es usada principalmente en el ambiento empresarial y educativo de manera que permita una comunicación simultánea con grandes grupos de trabajo. Por último, para las clases en línea se usa Microsoft Teams, que permite una colaboración de personas de un mismo equipo o institución compartiendo recursos y cuya función principal es la comunicación constante entre los miembros de la institución.

Últimamente, de las universidades de Quito se encontró que los profesores prefieren usar ZOOM y MICROSOFT TEAMS para desarrollar sus clases, que estás dos herramientas destacan entre muchas otras ya que cuentan con funciones didácticas e interactivas que ayudan a los estudiantes al aprendizaje y al desarrollo del razonamiento, estas funciones son: compartir pantalla, grabar pantalla, subir archivos mediante un chat compartido entre los miembros y muchas otras opciones con las que se puede combinar entre la clase.

\section{III.METODOLOGÍA}

Para esta investigación se ha escogido una metodología cuantitativa, por lo que se realizó una encuesta de 17 preguntas a 55 personas de la ciudad de Quito, que están relacionadas con la herramienta Microsoft Teams, las preguntas de la encuesta fueron evaluada dentro de un parámetro de menor importancia como la opción más baja dentro de un intervalo de 5 opciones siendo muy importante como la mayor calificación. Para la tabulación de los datos recopilados se usó el cálculo del Alfa de Cronbach, el cual nos permitió un análisis detallado de los datos, el cálculo nos arrojó una confiabilidad del 0.92, que dentro de los parámetros del cálculo es uno de los mejores ya que nos acredita el uso de la información.

El Alfa de Cronbach nos da una medida de la consistencia interna de una escala, que sus ítems apuntan en la misma dirección, entre las ventajas de esta medida se encuentra la posibilidad de evaluar cuanto mejoraría (o empeoraría) la fiabilidad de prueba si se excluyera un determinado ítem. Se utilizará el coeficiente Alfa de Cronbach para calcular la fiabilidad, salvo en casos en los que tengamos un interés expreso en conocer la consistencia entre dos o más partes de una prueba (por ej. primera mitad y segunda mitad; ítems pares e impares) o cuando queramos conocer otros "subtipos" de fiabilidad.

$$
\alpha=\frac{K}{K-1} \cdot\left|1-\frac{\sum V i}{V t}\right|
$$

\section{IV.RESULTADOS}

Entre los resultados, destacan los siguientes items, en cada figura se detallara cuales fueron las observaciones mas relevantes de este analisis.

Dentro de la evaluación se preguntó qué tan importante considera que el software sea una herramienta fácil de utilizar y oportuna para el aprendizaje, a las personas encuestadas se les dio 5 opciones de respuesta, donde la más baja era menos importante y la más alta muy importante, siendo para este ítem que $33 \%$ de la población cree que esta herramienta es muy importante y fácil para su aprendizaje, y solo el 7 \% de la población refleja una opinión de menor importancia es decir que se podría trabajar con otras herramientas para su aprendizaje. 


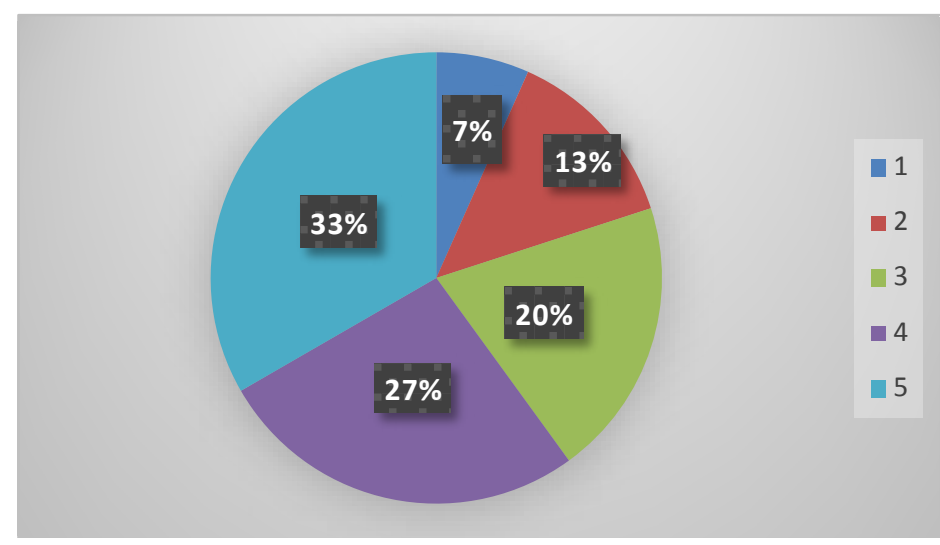

Fig.1 Evaluación de la importancia de la utilidad del software para el aprendizaje.

También se preguntó si se considera importante los símbolos utilizados por el software acorde a su función, uno de los principales problemas de las herramientas online es que muchas veces sus símbolos no concuerdan con su función y se puede mal interpretar cuando se presiona, para este ítem se obtuvo una respuesta de la población del $50 \%$ que no lo considera ni de baja importancia ni de mucha importancia.

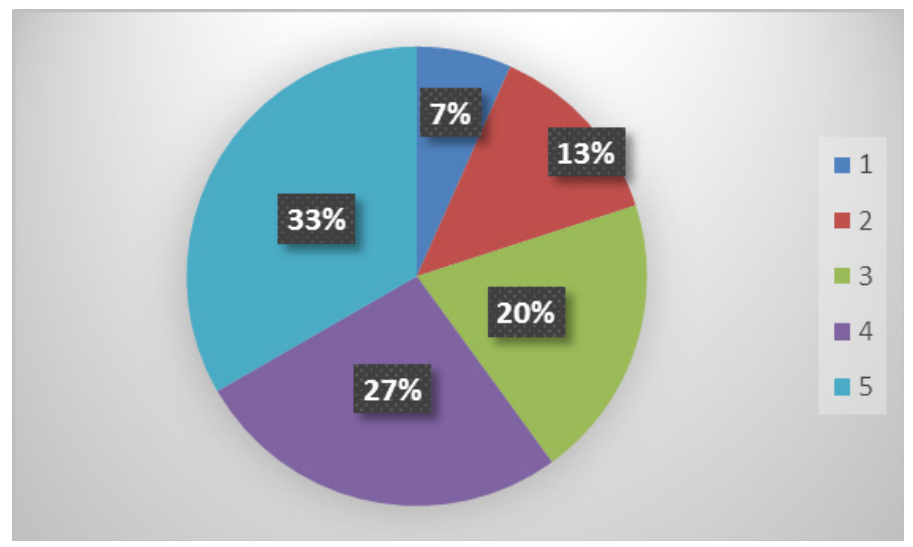

Fig.2 Evaluación de la importancia de los símbolos acorde a su función del software.

Además, se realizó dentro de la encuesta la pregunta del funcionamiento de las computadoras si ha servido como apoyo significativo en el aprendizaje, de un 100\% solo el $33 \%$ de los estudiantes piensan que para un aprendizaje integral y continuo se debe tener un computador que aporte con un buen funcionamiento.

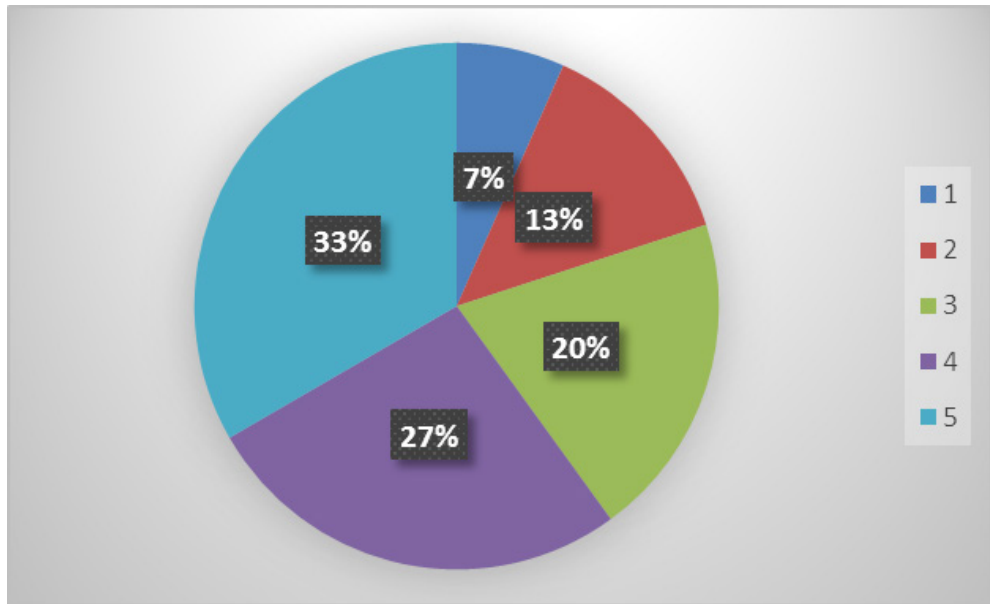

Fig.3 Evaluación de la importancia del funcionamiento de las computadoras para el aprendizaje con la herramienta 
Microsoft Teams.

Como no faltaba más se preguntó acerca de la importancia de los recursos de audio y video funcionen de manera rápida y eficaz dentro del software, se ha sabido desde siempre que el principal problema de las clases virtuales tiene el defecto de que los recursos de audio y video no funcionen ya se demore o no se cargue, este por supuesto es un análisis fundamental dentro de nuestra muestra que un $60 \%$ considera fundamental estas funciones en la herramienta Microsoft Teams.

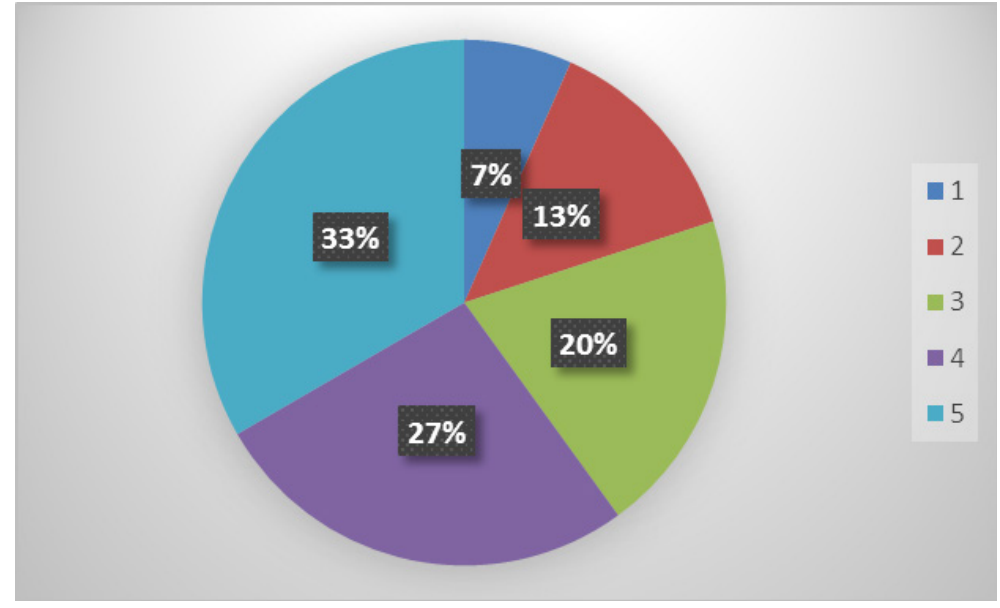

Fig.4 Evaluación de la importancia de los recursos de audio y video dentro del software.

\section{V.CONCLUSIONES}

Con este trabajo se ha podido analizar la satisfacción que tienen los estudiantes con el uso del software Microsoft Teams la cual ha sido indispensable para el aprendizaje online. Se puede concluir que, los datos y resultados obtenidos son de total confiabilidad gracias a la aplicación del alfa de Cronbach la cual arrojó un 0.92 lo que garantiza el manejo y procesamiento de datos, también se pude destacar, que la tecnología juega un papel fundamental dentro de la sociedad, ya que en este tiempo en el que nos encontramos en una pandemia la educación y el trabajo son aspectos que no pueden ser dejados de lado en nuestro país y la herramienta Microsoft Teams sin duda ha sido un software que ha contribuido con el desarrollo de la educación virtual en la Universidad de las Américas, para lo cual los estudiantes encuestados han respondido que es de vital importancia que la herramienta utilizada funcione de manera rápida y eficaz en el caso del audio y vídeo, obteniendo así un $60 \%$ que consideran fundamental que Microsoft Teams cumpla con este requisito. Las universidades prefieren de entre todas las herramientas disponibles la implementación de Teams y Zoom para su uso ya que estas poseen funciones didácticas e interactivas que ayudan a los estudiantes al aprendizaje y al desarrollo del razonamiento, estas funciones son compartir pantalla, grabar pantalla, subir archivos mediante un chat compartido entre los miembros y muchas otras opciones con las que se puede combinar entre la clase. En síntesis, "En la educación virtual el problema no es la herramienta, sino el uso que hacemos de ella" por lo que, se plantea la idea de escoger o de preferir herramientas colaborativas e interactivas lo que justifica quizá el contenido de la plataforma Microsoft Teams, llevando así la transformación de clases presenciales a un formato online calificando como aceptable las medidas tomadas por la institución.

\section{RECONOCIMIENTO}

Me van a faltar páginas para agradecer a las personas que se han involucrado en la realización de este trabajo, sin embargo, merecen reconocimiento especial nuestra Profesora Ing. Franyelit que con su dirección, esfuerzo y dedicación nos ayudó a culminar este proyecto de investigación y nos dio el apoyo suficiente para no decaer cuando todo parecía complicado e imposible. Asimismo, por sus comentarios de inspiración y aliento en el desarrollo de este proyecto que nos alentó a alcanzar nuevos objetivos y conocimientos.

\section{REFERENCIAS}

[1]C. E. F. Pabón, «MÁS ALLÁ DE ZOOM, GOOGLE MEET, TEAMS..., Slidesgo, vol. 1, nº 1, p. 12, 2020. 
[2]F. García, A. Correl, V. Abella y M. Grande, «La evaluación online en la educación superior en tiempos de la COVID-19,» EDUCATION IN THE KNOWLEDGE SOCIETY (EKS), vol. 21, nº 1, pp. 1-12, 2020.

[3]G. Jose, C. Alfredo, G. Victor y GrandeMario, «Education in the Knowledge Society,» Ediciones Universal Salamanca, vol. 1, n 12, p. 26, 14052020.

[4]E. Dans, «Educacion online: Plataformas educativas y el dilema de la apertura,» Universidad y Sociedad del Conocimiento, vol. 6, nº 1, p. 10, 02032009.

[5]A. Mauricio, A. Roberto, V. Edgar y F. Karen, «Convergencia de procesos de docencia universitaria: El uso de la aplicacion Teams de Microsoft,» ResearchGate, vol. 1, no 1, p. 9, 21112019.

[6]B. Jose, L. Lina, H. Maria y J. Eusebio, «Utilización del alfa de Cronbach para validar la confiabilidad,» Engineering and Technology, vol. 11, nº 1, p. 9, 16082013.

[7]J.-J. a. C.-R. D. a. D. P. Monedero-Moya, «Usabilidad y satisfacción en herramientas de anotaciones multimedia para MOOC $=$ Usability and Satisfaction in Multimedia Annotation Tools for MOOCs,» e-Lis, vol. 22, $\mathrm{n}^{\circ}$ 44, p. $62,2018$.

[8]I. Ros Martínez de Lahidalga, «Moodle, la plataforma para la enseñanza y organización escolar,» Ikastorratza, e- Revista de Didáctica, vol. 2, nº 50, p. 12, 2008.

[9]D. Marlene, «Herramientas para la producción de materiales didácticos para las modalidades de enseñanza semipresencial y a distancia,» ACIMED, vol. 16, nº 2, p. 5, 2007.

[10]C. Barbara, «Diseño y validación de un instrumento,» Universitat Illes Balears, vol. 4, nº 50, p. 415, 102006.

\section{RESUMEN CURRICULAR}

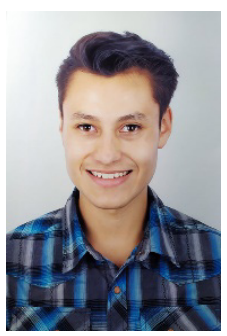

Mateo Sarauz, Estudiante de Ingeniería Industrial de la Universidad de las Américas, me gusta conocer nuevos lugares y adquirir experiencias inolvidables. Me gusta trabajar en equipo y pienso que la constancia es una virtud que todos debemos poseer para alcanzar nuestros objetivos y metas.

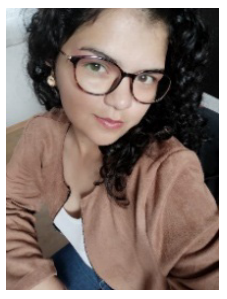

Rita Villafuerte, ecuatoriana de Nacimiento actualmente estudiante en la Universidad de las Américas en la carrera de Ing. Industrial, me considero una persona optimista, siempre con ganas de aprender y poner mis conocimientos al servicio de la comunidad.

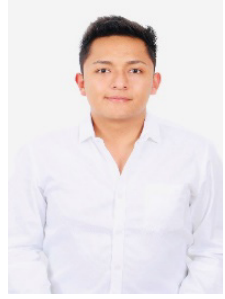

David Shuguli, me considero una persona responsable, dinámica y creativa, con la facilidad de adaptación y capacidad de trabajar en equipo, en condiciones de alta presión, con iniciativa para resolver problemas efectivamente y lograr las metas y objetivos trazados por la empresa.

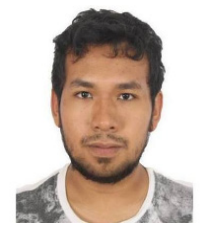

David Vaca, estoy estudiando una ingeniería en software en la Universidad de las américas me considero una amable, curiosa y creativas. Siendo uno de mis objetivos a largo plazo trabajar en la industria del desarrollo de software nacional o internacional de forma que expanda mis conocimientos y mi experiencia en el campo me permita seguir creciendo como ingeniero en software. 